\title{
Vasodilator $\beta$-blockers: a different class of antihypertensive agents?
}
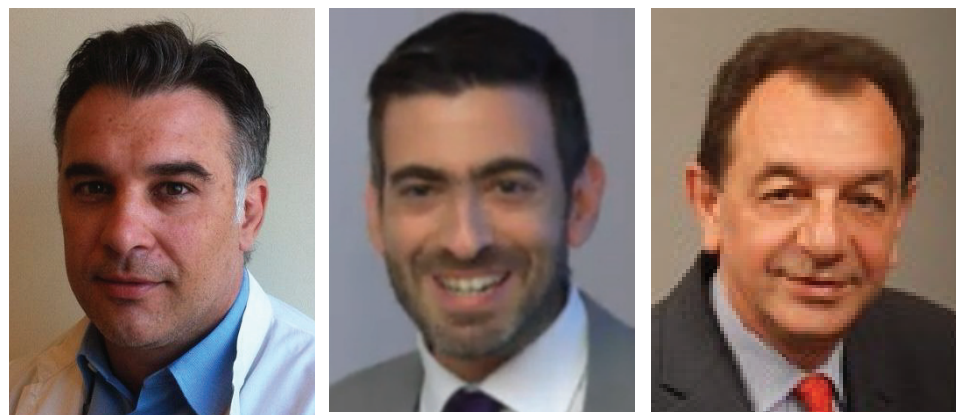

Manolis S Kallistratos ${ }^{*, 1}$, Leonidas E Poulimenos ${ }^{1} \&$ Athanasios J Manolis

$\beta$-blockers have been used for more than 40 years for the treatment of hypertension. Their initial acceptance in the early 1970s as a first-line antihypertensive drug class was mainly based on their excellent record of effectiveness and safety when compared with other available (old-fashioned) antihypertensive drugs at the time, or even to placebo. However, in the last few years, the use of these agents as a first-class approach for the treatment of hypertension, especially when it is uncomplicated, has been criticized by NICE and JNC8 guidelines, since their effect in a recent meta-analysis on cardiovascular (CV) morbidity and mortality was largely controversial [1]. $\beta$-blockers clearly reduce future $\mathrm{CV}$ events in patients who already have established heart disease, especially heart failure or early after a myocardial infarction; however, in primary prevention, these agents appear to be somewhat inferior to other antihypertensive drug classes currently in use. They tend to have more side effects [2], to be less effective in preventing organ damage (left ventricular hypertrophy, arterial stiffness, small artery remodeling and carotid intima media thickness) [3], while they probably accelerate the appearance of new-onset diabetes, impair the control of dyslipidemias and increase bodyweight [4]. Although current ESH/ESC guidelines suggest the use of these agents as first-line antihypertensive drugs based on several meta-analyses showing that $\beta$-blockers were practically equally effective with other major antihypertensive drug classes in preventing major cardiovascular events in younger or older hypertensive patients $[5,6]$ (however, they were less effective than calcium channel blockers from stroke, but not inferior to other drug classes [6]), the main issue for discussion is: should we continue to consider class effects in $\beta$-blockade? Are all $\beta$-blockers the same?

\section{Class effects of $\beta$-blockers: vasodilator $\beta$-blockers}

So far, authors and guidelines have considered the class effects of $\beta$-blockers, attributing advantages or disadvantages of these agents to all $\beta$-blockers. However, different $\beta$-blockers present different ancillary properties such as cardioselectivity, intrinsic sympathomimetic activity, lipophilicity and vasodilator properties. In particular, vasodilating $\beta$-blockers

\section{KEYWORDS}

- arterial hypertension $\bullet \beta$-blockers

- vasodilator $\beta$-blockers 


\section{"Vasodilator $\beta$-blockers exert a substantial BP reduction, with better metabolic profile and fewer side effects compared with older agents of the same class.”}

(carvedilol, nebivolol, celiprolol) seem to overcome the general limitations of the $\beta$-blockers mentioned above. These agents seem to have a better metabolic profile than other $\beta$-blockers, causing less insulin resistance, not worsening glucose tolerance, and having the potential to reduce central pulse pressure and aortic stiffness more than other compounds of the same class [7].

Carvedilol is a nonselective $\beta$-blocker, blocking concurrently $\alpha_{1}$ - and $\beta$-adrenergic receptors. Experimental and clinical trials showed that this agent promotes endothelial vasodilation, reduces platelet aggregation and inflammation, and preserves nitric oxide bioactivity [8-10]. In hypertensive diabetic patients, carvedilol seems to maintain glycemic control, while improving insulin sensitivity [11].

Nebivolol, on the other hand, has the highest $\mathrm{b}_{1}$-selectivity for human myocardium $\beta$-adrenergic receptors among the clinically used $\beta$-blockers [12]. It causes vasodilatation through activation of the L-arginine/nitric oxide pathway. Nebivolol has a favorable metabolic profile since it improves insulin sensitivity and does not cause deleterious effects on lipid profile $[13,14]$. In addition, it improves endothelial function and reduces arterial stiffness, mainly by stimulating endothelial nitric oxide release [15], while at the same time avoiding erectile dysfunction, a disturbing common side effect of $\beta$-adrenoceptor blockade [16].

Thus, a question arises: should we consider $\beta$-blockers as a single class of antihypertensive drugs or do we have to differentiate among $\beta$-blockers according to their specific properties? Until now, hypertension experts currently disagree on how to interpret the available data and this fact is reflected in current ESH/ESC [7] and JNC8 [17] guidelines, where the approach to consider $\beta$-blockers as a solid antihypertensive drug class differs.

\section{Studies on vasodilator $\beta$-blockers \& hypertension}

Although there is disagreement regarding the use of $\beta$-blockers in arterial hypertension, we have to agree that current evidence is mainly based on studies and meta-analyses of older $\beta$-blockers with no vasodilatatory properties, namely atenolol and metoprolol. Unfortunately, large studies with vasodilator $\beta$-blockers, especially in uncomplicated hypertensive populations, are largely missing.

Data on Celiprolol have mainly been derived from studies of patients with coronary artery disease (CAD), whereas data regarding carvedilol or nebivolol are from studies of patients with heart failure with or without CAD. In patients with arterial hypertension, data comes from small studies.

There are several studies affirming that 25-50 mg of carvedilol monotherapy leads to a consistent reduction in blood pressure (BP) over 24 h compared with placebo, and that carvedilol decreases arterial BP to a greater extent than atenolol in patients with essential hypertension [18]. In addition, comparative studies have shown that this agent reduced BP to a similar extent to other antihypertensive drugs, while combination of this drug with hydrochlorothiazide was well tolerated and further reduced BP levels [18].

On the other hand, there are also several studies with nebivolol versus placebo or versus the other major antihypertensive drug classes (including other $\beta$-blockers) where nebivolol was found to be effective in lowering BP [19]. However, massive data regarding this agent derived mainly from studies on patients with heart failure with reduced or preserved ejection fraction, where the addition of nebivolol led to a significant decrease of the composite end point of all-cause mortality or cardiovascular hospitalizations [20].

We have to keep in mind that according to the available scientific evidence by the megatrials and meta-analyses, the effect of antihypertensive drugs in patients with uncomplicated hypertension results from blood pressure reduction per se. The proportional reduction in major CV events, for a given reduction in blood pressure, is similar in hypertensive patients with or without a history of vascular disease [7]. Vasodilator $\beta$-blockers exert a substantial $\mathrm{BP}$ reduction, with better metabolic profile and fewer side effects compared with older agents of the same class.

\section{Conclusion}

Although data from large-scale studies assessing the effect of vasodilator $\beta$-blockers in patients with essential hypertension are missing, we have to keep in mind that only a small fraction of patients suffers from hypertension alone. The majority of hypertensive patients present overlapping risk factors and diseases and vasodilator $\beta$-blockers may have a significant role in this setting. In addition, vasodilator $\beta$-blockers have proven blood pressure-lowering effects and 
excellent safety and side effects profiles, both prerequisites for the benefits of any antihypertensive treatment.

\section{Financial \& competing interests disclosure}

The authors have no relevant affiliations or financial

involvement with any organization or entity with a financial interest in or financial conflict with the subject matter or materials discussed in the manuscript. This includes employment, consultancies, honoraria, stock ownership or options, expert testimony, grants or patents received or pending, or royalties.

No writing assistance was utilized in the production of this manuscript.

\section{References}

1 Wiysonge CS, Bradley HA, Volmink J, Mayosi BM, Mbewu A, Opie LH. Betablockers for hypertension. Cochrane Database Syst. Rev. 11, CD002003 (2012).

2 Silvestri A, Galetta P, Cerquetani E et al. Report of erectile dysfunction after therapy with betablockers is related to patient knowledge of side- effects and is reversed by placebo. Eur. Heart J. 24, 1928-1932 (2003).

3 Mancia G, Laurent S, Agabiti-Rosei E, Ambrosioni E, Burnier M, Caulfield MJ. Re-appraisal of European guidelines on hypertension management: a European Society of Hypertension Task Force document. J. Hypertens. 27, 2121-2158 (2009).

4 Elliott WJ, Meyer PM. Incident diabetes in clinical trials of antihypertensive drugs: a network meta-analysis. Lancet 369, 201-207 (2007).

5 Turnbull F1, Neal B, Algert C et al.; Blood Pressure Lowering Treatment Trialists' Collaboration. Effects of different blood pressure-lowering regimens on major cardiovascular events in individuals with and without diabetes mellitus: results of prospectively designed overviews of randomized trials. Arch. Intern. Med. 165, 1410-1419 (2005).

6 Law MR, Morris JK, Wald NJ. Use of blood pressure lowering drugs in the prevention of cardiovascular disease: meta-analysis of 147 randomised trials in the context of expectations from prospective epidemiological studies. BMJ 338, b1665 (2009).
7 Mancia G, Fagard R, Narkiewicz K et al. 2013 ESH/ESC guidelines for the management of arterial hypertension: the Task Force for the management of arterial hypertension of the European Society of Hypertension (ESH) and of the European Society of Cardiology (ESC). J. Hypertens. 31(7), 1281-1357 (2013).

8 Bristow MR. Beta-adrenergic receptor blockade in chronic heart failure. Circulation 101, 558-569 (2000).

9 Giugliano D, Marfella R, Acampora R, Giunta R, Coppola L, D'Onofrio F. Effects of perindopril and carvedilol on endotheliumdependent vascular functions in patients with diabetes and hypertension. Diabetes Care 21, 631-636 (1998).

10 Lopez BL, Christopher TA, Yue TL, Ruffolo R, Feuerstein GZ, Ma XL. Carvedilol, a new beta-adrenoreceptor blocker antihypertensive drug, protects against free-radical-induced endothelial dysfunction. Pharmacology 51, 165-173 (1995).

11 Bakris GL, Fonseca V, Katholi RE et al. Metabolic effects of carvedilol vs metoprolol in patients with Type 2 diabetes mellitus and hypertension: a randomized controlled trial. JAMA 292, 2227-2236 (2004).

12 Brixius K, Bundkirchen A, Bölck B. Nebivolol, bucindolol, metoprolol and carvedilol are devoid of intrinsic sympathomimetic activity in human myocardium. Br. J. Pharmacol. 133, 1330-1338 (2001).

13 Dhakam Z, Yasmin, McEniery CM. A comparison of atenolol and nebivolol in isolated systolic hypertension. J. Hypertens. 26(2), 351-356 (2008).
14 Lacourcière Y, Arnott W. Placebo-controlled comparison of the effects of nebivolol and low-dose hydrochlorothiazide as monotherapies and in combination on blood pressure and lipid profile in hypertensive patients. J. Hum. Hypertens. 8, 283-288 (1994).

15 Mason RP, Jacob RF, Corbalan JJ. The favourable kinetics and balance of nebivolol stimulated nitric oxide and peroxynitrite release in human endothelial cells. $B M C$ Pharmacol. Toxicol. 14, 48 (2013).

16 Brixius K, Middeke M, Lichtenthal A. Nitric oxide, erectile dysfunction and beta-blocker treatment (MR NOED study): benefit of nebivolol versus metoprolol in hypertensive men. Clin. Exp. Pharmacol. Physiol. 34, 327-331 (2007).

17 James PA, Oparil S, Carter BL et al. 2014 evidence-based guideline for the management of high blood pressure in adults: report from the panel members appointed to the Eighth Joint National Committee (JNC 8). JAMA 311(5), 507-520 (2014).

18 Leonetti G, Egan CG. Use of carvedilol in hypertension: an update. Vasc. Health Risk Manag. 8, 307-322 (2012).

19 Weiss RN. A novel beta-blocker with nitric oxide-induced vasodilatation. Vasc. Health Risk Manag. 2(3), 303-308 (2006).

20 Veldhuisen DJ, Cohen-Solal A, Böhm M et al. Beta-blockade with nebivolol in elderly heart failure patients with impaired and preserved left ventricular ejection fraction: data from SENIORS (Study of Effects of Nebivolol Intervention on Outcomes and Rehospitalization in Seniors with Heart Failure). J. Am. Coll. Cardiol. 53(23), 2150-2158 (2009). 